\title{
New Balance Capability Index as a Screening Tool for Mild Cognitive Impairment
}

\author{
Yasuhiro Suzuki \\ University of Tsukuba Hospital \\ Takumi Tsubaki \\ University of Tsukuba Hospital \\ Kensuke Nakaya \\ University of Tsukuba Hospital \\ Genta Kondo \\ University of Tsukuba \\ Yoshinori Takeuchi \\ University of Tsukuba \\ Yuichi Aita \\ University of Tsukuba \\ Yuki Murayama \\ University of Tsukuba
}

Akito Shikama

University of Tsukuba

Yukari Masuda

University of Tsukuba

Hiroaki Suzuki

University of Tsukuba

Yasushi Kawakami

University of Tsukuba

Hitoshi Shimano

University of Tsukuba

Tetsuaki Arai

University of Tsukuba

Yasushi Hada

University of Tsukuba Hospital

Naoya Yahagi ( $\nabla$ nyahagi-tky@umin.ac.jp )

University of Tsukuba 
Keywords: balance capability index, screening tool, mild cognitive impairment

Posted Date: October 28th, 2021

DOI: https://doi.org/10.21203/rs.3.rs-975835/v1

License: (c) (1) This work is licensed under a Creative Commons Attribution 4.0 International License. Read Full License 


\title{
New balance capability index as a screening tool for mild cognitive impairment
}

\author{
Yasuhiro Suzuki, ${ }^{1,2}$ Takumi Tsubaki, ${ }^{2}$ Kensuke Nakaya, ${ }^{2}$ Genta Kondo, ${ }^{1}$ \\ Yoshinori Takeuchi, ${ }^{3,4}$ Yuichi Aita, 3,4 Yuki Murayama, 3,4 Akito Shikama, 3,4 \\ Yukari Masuda, ${ }^{3}$ Hiroaki Suzuki, ${ }^{4}$ Yasushi Kawakami, ${ }^{5}$ Hitoshi Shimano, ${ }^{4}$ \\ Tetsuaki Arai, 6 Yasushi Hada, ${ }^{2}$ and Naoya Yahagi. $1,3,4,5^{*}$
}

From

SCORE (university promotion type) project team, Headquarters for International Industry-University Collaboration, University of Tsukuba, Ibaraki 305-8575, Japan ${ }^{1}$

Department of Rehabilitation Medicine, University of Tsukuba Hospital, Ibaraki

$$
\text { 305-8596, } \text { Japan }^{2}
$$

Nutrigenomics Research Group, Faculty of Medicine, University of Tsukuba, Ibaraki 305-8575, Japan 3

Department of Internal Medicine (Endocrinology and Metabolism), Faculty of Medicine, University of Tsukuba, Ibaraki 305-8575, Japan 4

Department of Laboratory Medicine, Faculty of Medicine, University of Tsukuba, Ibaraki 305-8575, Japan 5

Department of Psychiatry, Faculty of Medicine, University of Tsukuba, Ibaraki 305-8575, Japan6

*Address correspondence to:

Naoya Yahagi, MD., PhD.

1-1-1 Tennodai, Tsukuba, Ibaraki, Japan, 305-8575

E-mail: nyahagi-tky@umin.ac.jp 


\section{Abstract}

Mild cognitive impairment (MCI) is not just a prodrome to dementia, but a very important intervention point to treat Alzheimer's disease (AD). It has long been known that $\mathrm{AD}$ patients have a higher frequency of falls with some gait instability. Recent evidence suggests that vestibular impairment is disproportionately prevalent among individuals with MCI and dementia due to AD. Here we have developed a useful method to evaluate balance capability as well as vestibular function using Nintendo Wii balance board as a stabilometer and foam rubber on it. The new balance capability indicator, termed visual dependency index of postural stability (VPS), was highly associated with cognitive impairment assessed by MoCA, and the area under the receiver operating characteristic (ROC) curve was more than 0.8 , demonstrating high sensitivity and specificity. Thus, it was proved that VPS helps screen individuals with MCI at an early and preclinical stage with high sensitivity. 


\section{Introduction}

Approximately 50 million people live with dementia worldwide, and this number is predicted to increase to 152 million by $2050{ }^{1}$. Total payments in 2020 for people age 65 and older with dementia are already beyond $\$ 300$ billion only in the US ${ }^{2}$. Thus, the burden of dementia continues to grow, and prevention strategies are highly needed.

Although our understanding of dementia etiology is still shifting, Alzheimer's disease $(\mathrm{AD})$ is the most common type of dementia, accounting for an estimated $60 \%$ to $80 \%$ of cases ${ }^{2}$. The progression of $\mathrm{AD}$ is called the $\mathrm{AD}$ continuum. On this continuum, there are three broad phases: preclinical $\mathrm{AD}$, mild cognitive impairment (MCI) due to $\mathrm{AD}$ and dementia due to $\mathrm{AD}^{2}$. A meta-analysis study found that among individuals with MCI who were tracked for 5 years or longer, $38 \%$ developed dementia ${ }^{3}$. However, in some individuals MCI reverts to normal cognition or remains stable, particularly with some appropriate intervention ${ }^{1}$. Therefore, it is important to find individuals with MCI early in the course of $\mathrm{AD}$ in order to protect from dementia.

Generally, individuals with dementia are vulnerable for a decline in physical functioning and basic activities of daily living ${ }^{4}$. In fact, as a characteristics of cognitive 
impairment and dementia, it has long been known that $\mathrm{AD}$ patients have a higher frequency of falls ${ }^{5,6}$, and today, cognitive impairment is well established as an independent risk factor for falling ${ }^{7,8}$. Moreover, emerging evidence indicates that early disturbances in cognitive function are associated with slower gait and gait instability ${ }^{9,10}$. Given that gait and balance are deeply connected ${ }^{11}$, the close relationship between cognitive function and balance capability can be assumed.

The vestibular (inner ear balance) system senses head movement and orientation in space, and vestibular sensory input plays a critical role in postural control, contributing to balance capability. Intriguingly, growing evidence suggests that vestibular impairment is disproportionately prevalent among individuals with MCI and dementia due to $\mathrm{AD}^{12,13}$.

Posture in human is maintained using sensory inputs critical for balance, namely vestibular, visual, and somatosensory inputs ${ }^{14}$. Posturography is a technique used to quantify postural control in upright stance on a stabilometer. Recently, we and others have developed a useful method to evaluate balance capability as well as vestibular function using a stabilometer and foam rubber ${ }^{15-17}$. With further modifications to this method, here we report a new balance capability indicator, named visual dependency index of postural stability (VPS), which is highly associated with 
cognitive impairment and helps screen individuals with MCI. 


\section{Results}

\section{Wii balance board (WBB) has the same performance as an authentic stabilometer}

Because the final goal of our project is to implement an inexpensive MCI screening system to prevent dementia, we started from developing a new posturographic system utilizing Wii balance board (WBB), a Nintendo game machine available at a very low cost (lower than $\$ 100$ ). According to several previous reports where WBB was tested in a clinical setting to track and record the center of pressure (COP) of subjects ${ }^{18-20}$, we developed a new software run on windows PC to measure and calculate index of postural stability (IPS) values (Figure 1A) ${ }^{15,17}$. As expected, WBB showed an excellent performance to measure IPS values as accurately as an authentic stabilometer for medical use (GP-6000 gravicorder), as shown in Figure 1B.

\section{VPS and cognitive function show a significant relationship}

Next, we devised a new indicator of postural stability that is more associated with vestibular function, based on the previous reports: combination of IPS ${ }^{15}$ and utilization of foam rubber as well as eye closure $16,17,21$. The resultant new indicator, named visual dependency index of postural stability (VPS), was defined as the ratio of 
eye-opened $v s$. eye-closed IPS values measured on foam rubber.

Using VPS, the new indicator of vestibular function calculated from balance measurement, we examined the possible association between vestibular function and cognitive impairment. Table 1 showed the clinical characteristics of the study participants, basically healthy volunteers $(n=49)$. Japanese version of the Montreal Cognitive Assessment (MoCA) was used to assess cognitive function ${ }^{22,23}$. It is widely accepted that MoCA is superior to MMSE in the detection of MCI as the MMSE has lower sensitivity ${ }^{23,24}$. In fact, as shown in Table 2, both the normal and MCI groups diagnosed based on MoCA showed nearly full score (30 points) on MMSE. As shown in Figure 2A, VPS values exhibited a significant negative correlation with MoCA scores. Also, significant association was observed between VPS and TMT-B scores, another scoring method to evaluate cognitive function (Figure $2 \mathrm{~B}, \mathrm{C}$ ).

\section{MCI group has significantly higher VPS}

In accordance with the above result, when participants were categorized into two groups of normal (MoCA $\geq 26)$ and $\mathrm{MCI}(\mathrm{MoCA} \leq 25)$, MCI group showed significantly higher VPS values (Figure 3A), despite no differences in IPS values between the groups (Figure 3B). All the other items were not significantly different 
between the two groups (Table 1).

\section{ROC curve shows good sensitivity and specificity}

The result of the receiver operating characteristic (ROC) analysis was shown in Figure 4. As shown there, area under of the curve (AUC) was more than 0.8, indicating a good sensitivity and specificity in general. The VPS cutoff value obtained from the maximum value of the Youden Index was 1.00 (sensitivity; $81.3 \%$, specificity: $57.6 \%$, $95 \%$ confidential interval: $0.675-0.936)$. In other words, if it exceeds the cutoff value of 1.00 , it is highly likely that it is MCI, and conversely, if it is below it, it is highly likely that it is not MCI. 


\section{Discussion}

In the present study, we clearly demonstrated that our new balance indicator

VPS is useful to screen individuals with MCI with high sensitivity.

The new indicator VPS (visual dependency index of postural stability) was created from the combination of IPS (index of postural stability) quantification method 15,17 and foam posturography technique ${ }^{16}$, both established previously. Among three sensory inputs critical for balance, namely vestibular, visual, and somatosensory inputs 14, the usage of foam rubber disrupts the somatosensory input, and eye closure additionally eliminates visual input. Therefore, it is assumed that vestibular function can easily be assessed on foam rubber with eye-closed condition ${ }^{16}$. Although we have not checked the point by ourselves, they examined the relationship between foam posturography data and direct vestibular function tests using cervical vestibular evoked myogenic potentials (cVEMPs), a well-established clinical test to examine vestibular function, and concluded that foam posturography on an eye-closed condition was useful for assessing vestibular impairment with abnormal cVEMPs ${ }^{21}$. Further taking the ratio between eye-closed and -opened conditions, we managed to raise the accuracy of measurement and named the value VPS. 
Regarding the relationship between vestibular dysfunction and cognitive impairment, emerging evidence suggests that vestibular loss is disproportionately prevalent among individuals with $\mathrm{MCI}$ and dementia due to $\mathrm{AD}{ }^{12,13,25}$; in a study named Baltimore Longitudinal Study of Aging (BLSA), testing 183 healthy community-dwelling participants with a mean age of 72 , they found that poorer vestibular function was significantly associated with poorer cognitive function assessed by several testing including TMT-B ${ }^{12}$. In addition, it has previously been shown that vestibular loss causes hippocampal atrophy and impaired spatial memory in humans ${ }^{26}$. A further study in BLSA found that poorer vestibular function was associated with significantly reduced hippocampal volume ${ }^{27}$. Thus, hippocampal atrophy may underlie the link between vestibular loss and cognitive decline.

Related to the link between vestibular and cognitive functions, hearing impairment is also reported to be linked with cognitive decline; a small US prospective cohort study of 194 adults without baseline cognitive impairment and at least two brain MRIs with a mean of 19 years follow-up, found that midlife hearing impairment measured by audiometry is associated with steeper temporal lobe volume loss, including in the hippocampus and entorhinal cortex ${ }^{28}$. The positive association between the loss of inner ear function and cognitive impairment is noteworthy. 
Considering further in this regard, inner ear dysfunction and hippocampal atrophy might have some underlying pathological processes shared in common. The hippocampus has been known as one of the limited areas in the adult mammalian brain where neurogenesis normally occurs ${ }^{29}$, and it is widely acknowledged that hippocampal neurogenesis is impaired in $\mathrm{AD}$, which plays a role in cognitive decline ${ }^{30,31}$. On the other hand, recent evidence suggests that reactive adult neurogenesis occurs in sensory systems following damages to the sensory nerve, and in fact this mechanism promotes balance recovery after vestibular loss ${ }^{32}$. Thus, it is conceivable that some common disorder related to neurogenesis in $\mathrm{AD}$ might underlie the link between inner ear dysfunction and hippocampal atrophy. Although currently this is just a hypothesis and further investigation is needed to prove it, this hypothesis might be related to the fact that $\mathrm{AD}$ is also strongly associated with olfactory dysfunction ${ }^{33-36}$, given that olfactory bulb is another area of the adult brain where neurogenesis occurs vigorously ${ }^{29}$ and patients with AD exhibit smaller olfactory bulb volumes ${ }^{37}$.

MCI is not just a prodrome to dementia, but a very important intervention point to treat $\mathrm{AD}$. Recently, aducanumab, an antibody drug targeting $\mathrm{A} \beta^{38}$, was approved by FDA in the US ${ }^{39}$. This decision made aducanumab the first new drug to be approved for the treatment of $\mathrm{AD}$ since 2003 and the first drug to ever be approved for modification 
of the course of $\mathrm{AD}$. Since the drug is targeting MCI and early AD, the demand for efficient screening of MCI patients will become larger in the near future.

Because we started this project to realize an inexpensive and easy-to-use MCI screening system, we focused on WBB, a very popular Nintendo game machine, and successfully proved that it can be used as an excellent stabilometer that has the same performance as an authentic apparatus approved for the medical use. Nintendo has so far sold out more than 37 million WBB worldwide (https://www.nintendo.co.jp/ir/pdf/2009/091030.pdf). It means a huge potential for the novel approach to diagnose MCI at an earlier stage.

In summary, we developed a new balance capability index termed VPS using WBB as a stabilometer. This method is useful to screen individuals with MCI at an early and preclinical stage with high sensitivity. 


\section{Methods}

Participants - From December 2020 to February 2021, we enrolled 49 participants with healthy conditions who were 56 to 75 years old and had no clinically apparent cognitive impairment. The participants were recruited using our department's website, bulletin boards at our university, and a local community magazine. Participants were paid a reward of 7,000 yen. Exclusion criteria included (i) history of chronic diseases (dementia, diabetes, kidney disease, collagen disease, peripheral neuropathy); (ii) Having a disability certificate or using long-term care insurance; (iii) being unable to walk independently without assistive devices (including transportation). No one was excluded based on results of cognitive function tests. Written informed consent was obtained from participants prior to study enrollment. This study was conducted in accordance with the principles of the Declaration of Helsinki and approved by the Clinical Research Ethics Committee of University of Tsukuba Hospital (R02-251).

Clinical Evaluation - Participants were surveyed for age, sex, exercise habitation, alcohol drinking habitation, working, driving. Body composition was evaluated by bioelectrical impedance analysis (InBody 720, BioSpace, Tokyo). Body mass index 
(BMI) and skeletal muscle mass index were calculated by dividing the body weight $(\mathrm{kg})$ by the square of the height $\left(\mathrm{m}^{2}\right)$ and dividing the limb skeletal muscle mass $(\mathrm{kg})$ by the square of the height $\left(\mathrm{m}^{2}\right)$, respectively.

Assessment of Cognitive Function - Global cognitive function was assessed using the Japanese version of the Montreal Cognitive Assessment (MoCA) ${ }^{22,23}$ and the Mini-Mental State Examination (MMSE) ${ }^{40}$. Based on MoCA, participants were categorized into two groups of normal $(\mathrm{MoCA} \geq 26)$ and $\mathrm{MCI}(\mathrm{MoCA} \leq 25)$. Trail Making Test parts A and B (TMT-A/-B) were used for assessing processing speed and executive function, respectively ${ }^{41}$. Clinical dementia rating (CDR) were used as an observational scale to assess the severity of dementia ${ }^{42-44}$.

Assessment of Balance capabilities - Balance capabilities were assessed by the index of postural stability (IPS) and the visual dependency index of postural stability (VPS). IPS and VPS were measured using a stabilometer as described previously ${ }^{17}$. As a stabilometer, GP-6000 gravicorder (Anima Co., Tokyo, Japan) and Wii balance board (WBB; Nintendo Co, Kyoto, Japan) were used. Briefly, IPS was measured as follows; first, the participants stood in a resting position with the inside of the foot at a distance 
of $10 \mathrm{~cm}$ on the stabilometer to measure the instantaneous fluctuations in the center of pressure (COP). Then, participants were instructed to incline the body to the front, rear, right and left keeping the body straight and without moving the feet for 10 seconds at each position, and the instantaneous fluctuations in COP were recorded. IPS was calculated as $\log [($ area of stability limit + area of postural sway $) /$ area of postural sway]. Area of stability limit was calculated as the front and rear center movement distance between anterior and posterior positions $\times$ the distance between right and left positions. Area of postural sway was calculated as average measurement value in 10 seconds under anterior, posterior, right, left and center positions. The area of postural sway was calculated as the mean sway area of the 5 positions. Visual dependency index of postural stability (VPS) was defined as the ratio of eye-opened vs. eye-closed IPS values measured on foam rubber. VPS values are age-adjusted by the following formula: age-adjusted VPS $=\mathrm{V} / \mathrm{V}^{\prime}$, where

$\mathrm{V}($ measured VPS $)=$ eye-opened IPS / eye-closed IPS, V' (age-predicted VPS $)=\mathrm{O} / \mathrm{C}$,

$\mathrm{O}$ (age-predicted eye-opened IPS) $=-0.0003 \mathrm{x} \mathrm{age}^{2}+0.0145 \mathrm{x}$ age +1.1602 (Supplementary Figure S1) and

$\mathrm{C}$ (age-predicted eye-closed IPS) $=-0.00006 \times$ age $^{2}-0.0037 \times$ age +0.8805 (Supplementary Figure S2). 
For a measurement using WBB, WBB was connected wirelessly with a Bluetooth adapter to a laptop computer. Raw data were collected simultaneously, stored and processed using custom-written software (Penguin system Co, Ibaraki, Japan).

Statistical analyses - Based on distribution, continuous variables were expressed as deviation or median (interquartile range) and compared using the unpaired -test or the Mann-Whitney -test for two-group comparisons. Categorical variables are expressed as numerals and percentages and were compared with Fisher's exact test. Spearman's rank correlation coefficient was used to examine bivariate associations between tests of cognitive function and balance capability. Pearson's correlation coefficient was used to examine bivariate associations between IPS of the GP-6000 gravicorder and that of WBB. Regarding the receiver operating characteristic (ROC) analysis, the VPS value and 01 classification ( 0 for Normal group, 1 for MCI group) for the presence or absence of MCI, the cutoff value, sensitivity, specificity, and area under of the curve (AUC) for the presence or absence of MCI were calculated. The cutoff value was decided by the point where the Youdem Index reaches the maximum value. Statistical analyses were performed using SPSS Statistics 26 (Chicago, IL, USA). Statistical significance was considered at a value of $<0.05$. 


\section{References}

1. Livingston, G. et al. Dementia prevention, intervention, and care: 2020 report of the Lancet Commission. Lancet 396, 413-446 (2020).

2. (No authors listed) 2020 Alzheimer's disease facts and figures. Alzheimers Dement (2020).

3. Mitchell, A. J. \& Shiri-Feshki, M. Rate of progression of mild cognitive impairment to dementia--meta-analysis of 41 robust inception cohort studies. Acta Psychiatr Scand 119, 252-65 (2009).

4. Blankevoort, C. G. et al. Review of effects of physical activity on strength, balance, mobility and ADL performance in elderly subjects with dementia. Dement Geriatr Cogn Disord 30, 392-402 (2010).

5. Morris, J. C., Rubin, E. H., Morris, E. J. \& Mandel, S. A. Senile dementia of the Alzheimer's type: an important risk factor for serious falls. J Gerontol 42, 412-7 
(1987).

6. Buchner, D. M. \& Larson, E. B. Falls and fractures in patients with Alzheimer-type dementia. Jama 257, 1492-5 (1987).

7. van Doorn, C. et al. Dementia as a risk factor for falls and fall injuries among nursing home residents. J Am Geriatr Soc 51, 1213-8 (2003).

8. Muir, S. W., Gopaul, K. \& Montero Odasso, M. M. The role of cognitive impairment in fall risk among older adults: a systematic review and meta-analysis. Age Ageing 41, 299-308 (2012).

9. Montero-Odasso, M., Verghese, J., Beauchet, O. \& Hausdorff, J. M. Gait and cognition: a complementary approach to understanding brain function and the risk of falling. J Am Geriatr Soc 60, 2127-36 (2012).

10. Bahureksa, L. et al. The Impact of Mild Cognitive Impairment on Gait and Balance: A Systematic Review and Meta-Analysis of Studies Using 
Instrumented Assessment. Gerontology 63, 67-83 (2017).

11. Visser, H. Gait and balance in senile dementia of Alzheimer's type. Age Ageing 12, 296-301 (1983).

12. Bigelow, R. T. et al. Association Between Visuospatial Ability and Vestibular Function in the Baltimore Longitudinal Study of Aging. J Am Geriatr Soc 63, $1837-44(2015)$.

13. Agrawal, Y., Smith, P. F. \& Rosenberg, P. B. Vestibular impairment, cognitive decline and Alzheimer's disease: balancing the evidence. Aging Ment Health 24, 705-708 (2020).

14. Furman, J. M. Role of posturography in the management of vestibular patients. Otolaryngol Head Neck Surg 112, 8-15 (1995).

15. Mochizuki, H. \& Mineshima, T. Reliability and validity of the Index of Postural Stability using forceplates. Rigaku Ryoho Gaku 27, 199-203 (2000). 
16. Fujimoto, C. et al. Assessment of diagnostic accuracy of foam posturography for peripheral vestibular disorders: analysis of parameters related to visual and somatosensory dependence. Clin Neurophysiol 120, 1408-14 (2009).

17. Suzuki, Y. et al. Age-dependent changes in dynamic standing-balance ability evaluated quantitatively using a stabilometer. J Phys Ther Sci 30, 86-91 (2018).

18. Clark, R. A. et al. Validity and reliability of the Nintendo Wii Balance Board for assessment of standing balance. Gait Posture 31, 307-10 (2010).

19. Park, D. S. \& Lee, G. Validity and reliability of balance assessment software using the Nintendo Wii balance board: usability and validation. J Neuroeng Rehabil 11, 99 (2014).

20. Leach, J. M., Mancini, M., Peterka, R. J., Hayes, T. L. \& Horak, F. B. Validating and calibrating the Nintendo Wii balance board to derive reliable center of pressure measures. Sensors (Basel) 14, 18244-67 (2014). 
21. Fujimoto, C. et al. Effects of unilateral dysfunction of the inferior vestibular nerve system on postural stability. Clin Neurophysiol 121, 1279-84 (2010).

22. Nasreddine, Z. S. et al. The Montreal Cognitive Assessment, MoCA: a brief screening tool for mild cognitive impairment. J Am Geriatr Soc 53, 695-9 (2005).

23. Fujiwara, Y. et al. Brief screening tool for mild cognitive impairment in older Japanese: validation of the Japanese version of the Montreal Cognitive Assessment. Geriatr Gerontol Int 10, 225-32 (2010).

24. Breton, A., Casey, D. \& Arnaoutoglou, N. A. Cognitive tests for the detection of mild cognitive impairment (MCI), the prodromal stage of dementia: Meta-analysis of diagnostic accuracy studies. Int J Geriatr Psychiatry 34, 233-242 (2019).

25. Dobbels, B. et al. Impact of Bilateral Vestibulopathy on Spatial and Nonspatial 
Cognition: A Systematic Review. Ear Hear 40, 757-765 (2019).

26. Brandt, T. et al. Vestibular loss causes hippocampal atrophy and impaired spatial memory in humans. Brain 128, 2732-41 (2005).

27. Kamil, R. J., Jacob, A., Ratnanather, J. T., Resnick, S. M. \& Agrawal, Y.

Vestibular Function and Hippocampal Volume in the Baltimore Longitudinal Study of Aging (BLSA). Otol Neurotol 39, 765-771 (2018).

28. Armstrong, N. M. et al. Association of Midlife Hearing Impairment With Late-Life Temporal Lobe Volume Loss. JAMA Otolaryngol Head Neck Surg 145, 794-802 (2019).

29. Magavi, S. S., Leavitt, B. R. \& Macklis, J. D. Induction of neurogenesis in the neocortex of adult mice. Nature 405, 951-5 (2000).

30. Rodriguez, J. J. et al. Impaired adult neurogenesis in the dentate gyrus of a triple transgenic mouse model of Alzheimer's disease. PLoS One 3, e2935 (2008). 
31. Lazarov, O. \& Hollands, C. Hippocampal neurogenesis: Learning to remember. Prog Neurobiol 138-140, 1-18 (2016).

32. Tighilet, B. \& Chabbert, C. Adult neurogenesis promotes balance recovery after vestibular loss. Prog Neurobiol 174, 28-35 (2019).

33. Wilson, R. S., Arnold, S. E., Schneider, J. A., Tang, Y. \& Bennett, D. A. The relationship between cerebral Alzheimer's disease pathology and odour identification in old age. J Neurol Neurosurg Psychiatry 78, 30-5 (2007).

34. Wesson, D. W., Levy, E., Nixon, R. A. \& Wilson, D. A. Olfactory dysfunction correlates with amyloid-beta burden in an Alzheimer's disease mouse model. $J$ Neurosci 30, 505-14 (2010).

35. Kotecha, A. M., Correa, A. D. C., Fisher, K. M. \& Rushworth, J. V. Olfactory Dysfunction as a Global Biomarker for Sniffing out Alzheimer's Disease: A Meta-Analysis. Biosensors (Basel) 8 (2018). 
36. Jung, H. J., Shin, I. S. \& Lee, J. E. Olfactory function in mild cognitive impairment and Alzheimer's disease: A meta-analysis. Laryngoscope 129, $362-369$ (2019).

37. Jobin, B., Boller, B. \& Frasnelli, J. Volumetry of Olfactory Structures in Mild Cognitive Impairment and Alzheimer's Disease: A Systematic Review and a Meta-Analysis. Brain Sci 11 (2021).

38. Sevigny, J. et al. The antibody aducanumab reduces Abeta plaques in Alzheimer's disease. Nature 537, 50-6 (2016).

39. Mullard, A. FDA approval for Biogen's aducanumab sparks Alzheimer disease firestorm. Nat Rev Drug Discov 20, 496 (2021).

40. Folstein, M. F., Folstein, S. E. \& McHugh, P. R. "Mini-mental state". A practical method for grading the cognitive state of patients for the clinician. $J$ Psychiatr Res 12, 189-98 (1975). 
41. Bowie, C. R. \& Harvey, P. D. Administration and interpretation of the Trail Making Test. Nat Protoc 1, 2277-81 (2006).

42. Hughes, C. P., Berg, L., Danziger, W. L., Coben, L. A. \& Martin, R. L. A new clinical scale for the staging of dementia. Br J Psychiatry 140, 566-72 (1982).

43. Gelb, D. J. \& St Laurent, R. T. Alternative calculation of the global clinical dementia rating. Alzheimer Dis Assoc Disord 7, 202-11 (1993).

44. Morris, J. C. The Clinical Dementia Rating (CDR): current version and scoring rules. Neurology 43, 2412-4 (1993). 


\section{Acknowledgements}

We thank all the SCORE project members at Headquarters for International Industry-University Collaboration, University of Tsukuba, for continuous support for the project. We also thank Ibaraki Occupational Therapist Association for helping to evaluate participants cognitive function. We also thank Dr. Yukio Yanagisawa at Department of Physical Therapy, Faculty of Health and Welfare, Tokushima Bunri University, for giving us valuable advice. This work was financially supported by Japan Science and Technology Agency (JST) SCORE (Program of Start-up incubation from COre REsearch) program (university promotion type).

\section{Author contributions}

Y.S. and N.Y. conceived the study. Y.S. T.T. and K.N. performed the study and analyzed the data together with N.Y. Y.S. and N.Y. co-wrote the paper. All authors discussed the results and commented on the manuscript.

\section{Competing interests}

The authors declare no competing financial and non-financial interests. 


\section{Figure legends}

Figure 1. WBB has the same performance as an authentic stabilometer.

(A) Schematic representation of the IPS measurement system using WBB.

(B) Correlation between IPS values measured by an authentic stabilometer (GP-6000 gravicorder) and those by WBB is shown (n=49). IPS, index of postural stability; VPS, visual dependency index of postural stability; WBB, Wii balance board.

Figure 2. VPS and cognitive function show a significant relationship.

Correlation between test VPS and cognitive function is shown. Cognitive function is assessed by (A) MoCA, (B) TMT-A and (C) TMT-B. VPS, visual dependency index of postural stability; MoCA, Montreal cognitive assessment; TMT-A, trail making test part A; TMT-B, trail making test part B.

Figure 3. MCI group has significantly higher VPS.

Comparison of the normal group and the MCI group is shown. Data are analyzed by the Un paired T-test. ${ }^{*} P<0.01$. MCI, mild cognitive impairment; IPS, index of postural stability; VPS, visual dependency index of postural stability. 
Figure 4. ROC curve shows good sensitivity and specificity.

ROC curve of VPS is drawn to discriminate MCI from normal. ROC, receiver operating characteristic; VPS, visual dependency index of postural stability; MCI, mild cognitive impairment; AUC, area under the curve. 


\section{Figures}

\section{Figure 1}

A

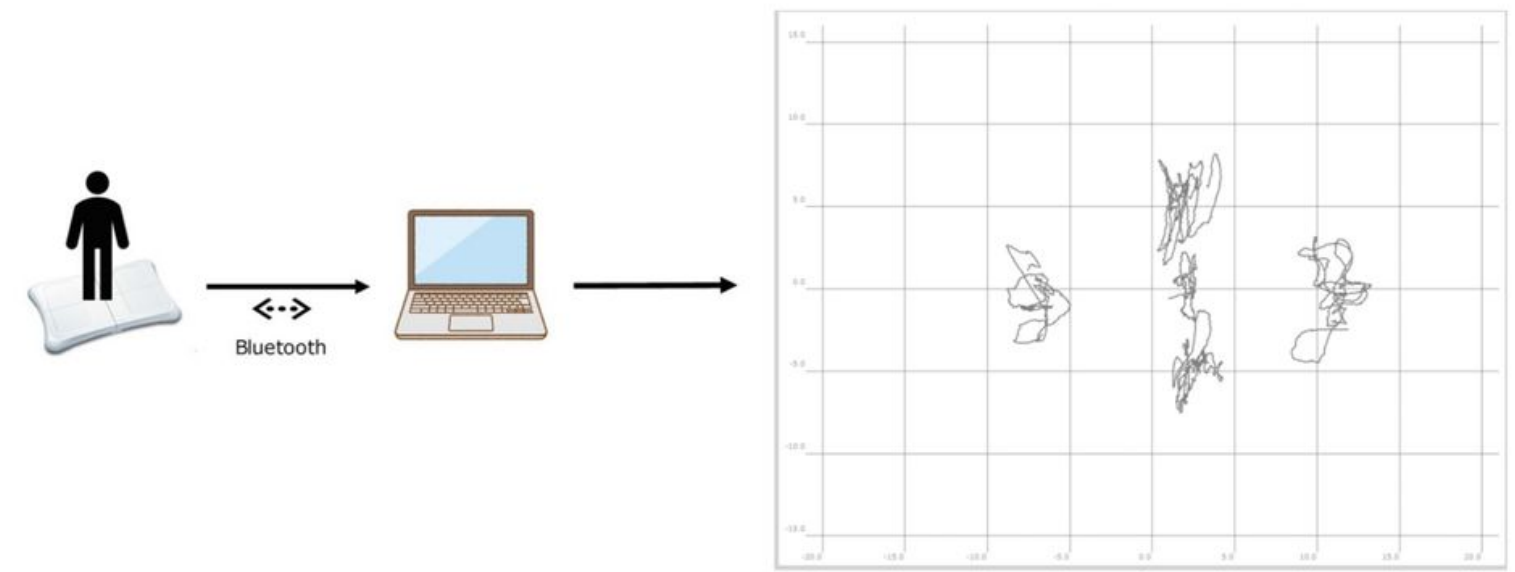

B

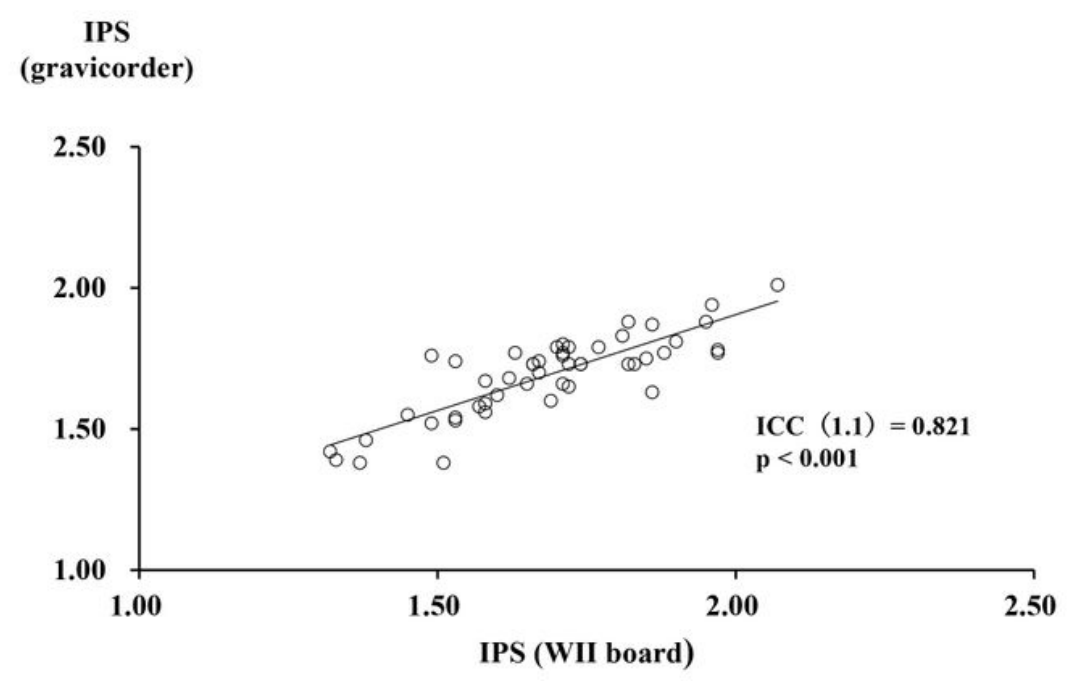

\section{Figure 1}

WBB has the same performance as an authentic stabilometer. (A) Schematic representation of the IPS measurement system using WBB. (B) Correlation between IPS values measured by an authentic stabilometer (GP-6000 gravicorder) and those by WBB is shown ( $n=49)$. IPS, index of postural stability; VPS, visual dependency index of postural stability; WBB, Wii balance board. 
Figure 2
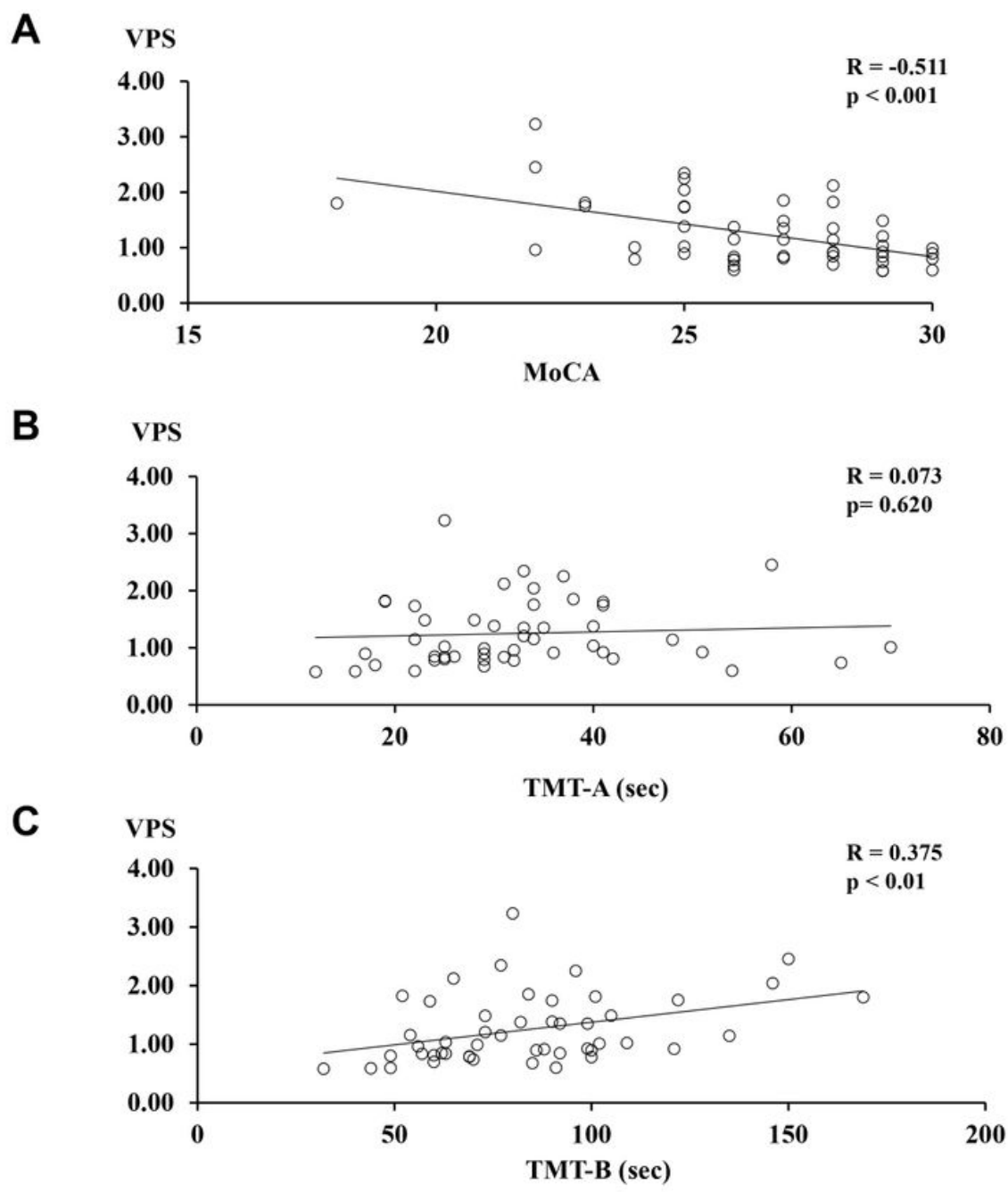

Figure 2

VPS and cognitive function show a significant relationship. Correlation between test VPS and cognitive function is shown. Cognitive function is assessed by (A) MoCA, (B) TMT-A and (C) TMT-B. VPS, visual dependency index of postural stability; MoCA, Montreal cognitive assessment; TMT-A, trail making test part A; TMT-B, trail making test part B. 
Figure 3

A
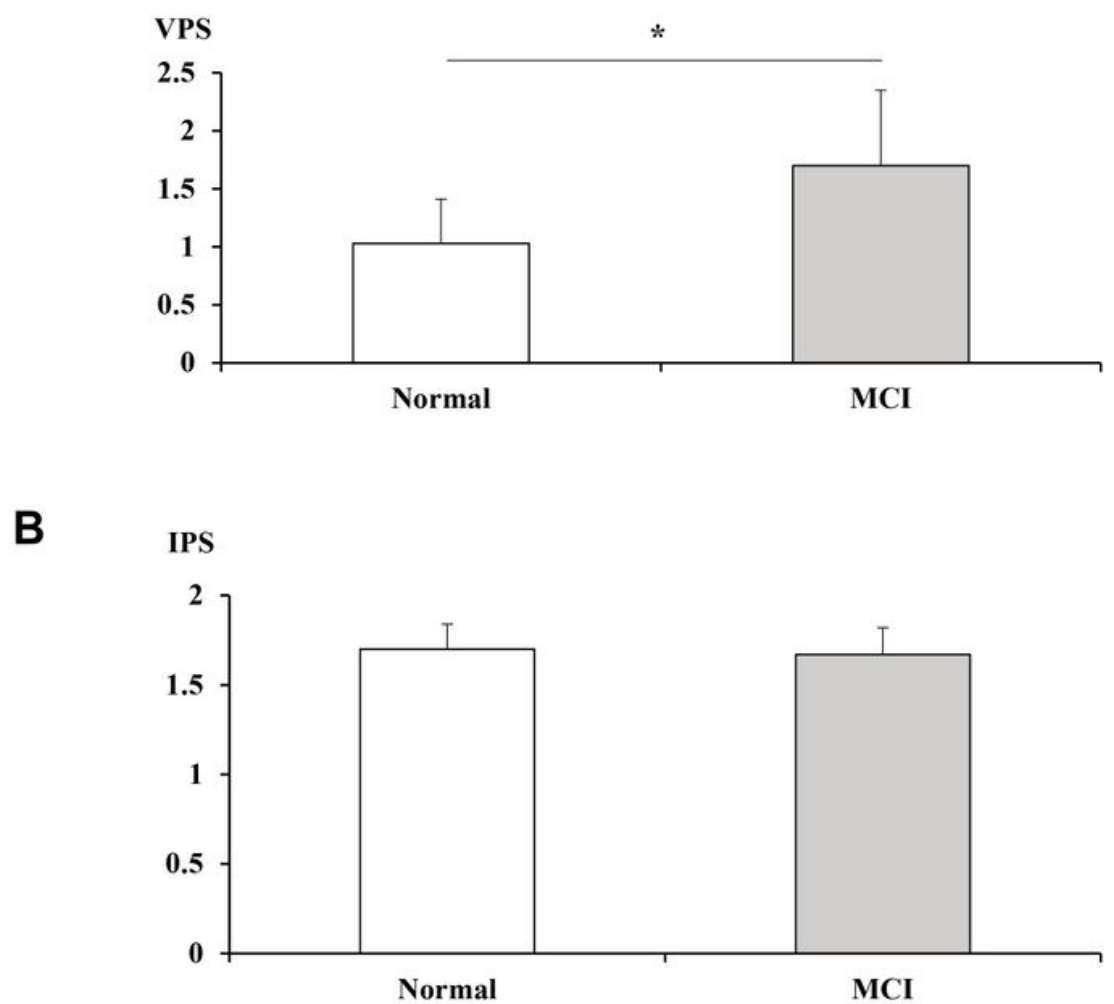

Figure 3

$\mathrm{MCl}$ group has significantly higher VPS. Comparison of the normal group and the $\mathrm{MCl}$ group is shown. Data are analyzed by the Un paired T-test. ${ }^{*} \mathrm{P}<0.01$. MCl, mild cognitive impairment; IPS, index of postural stability; VPS, visual dependency index of postural stability 


\section{Figure 4}

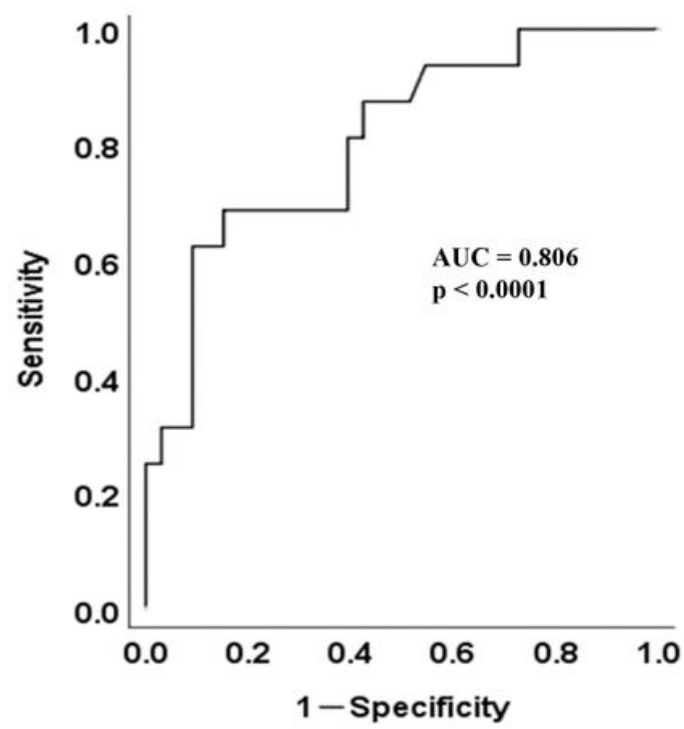

\section{Figure 4}

ROC curve shows good sensitivity and specificity. ROC curve of VPS is drawn to discriminate $\mathrm{MCl}$ from normal. ROC, receiver operating characteristic; VPS, visual dependency index of postural stability; $\mathrm{MCl}$, mild cognitive impairment; AUC, area under the curve

\section{Supplementary Files}

This is a list of supplementary files associated with this preprint. Click to download.

- Supplementarylnformation.pdf 